\title{
EUTANASIA PARA MENORES DE EDAD EN COLOMBIA, DILEMAS ÉTICOS Y JURÍDICOS DE LA MUERTE DIGNA EN NIÑOS, NIÑAS Y ADOLESCENTES
}

\author{
DAYRON REYES \& GABRIELA SUÁREZ
}

\author{
Abogados, Universidad Francisco de Paula Santander Ocaña - Colombia \\ ddreyesq@ufpso.edu.co
}

\begin{abstract}
RESUMEN: El presente artículo busca comprender un tema que suscita discusiones interminables dentro de la sociedad: la eutanasia. Hasta ahora solo se pensaba que el derecho a morir dignamente en Colombia le pertenecía a los mayores de edad; sin embargo, por mandato de la Corte Constitucional, se ha abierto la oportunidad para que menores de edad accedan a este procedimiento. Colombia es el tercer país del mundo en establecer un trámite para la eutanasia en menores de edad, pero esta regulación ha despertado serias dudas y ha avivado el debate acerca de las implicaciones éticas, sociales y jurídicas de la eutanasia.
\end{abstract}

PALABRAS CLAVE: eutanasia, cuidados paliativos, muerte digna, enfermo terminal, Corte Constitucional, autonomía de los menores.

ABSTRACT: This article seeks to understand Euthanasia as a topic that raises endless discussions in our society. Until now, the right to die with dignity was only thought for those who were of legal age in Colombia; however, by order of the Constitutional Court, this opportunity has been opened for minors to access it. Colombia is the third country in the world to establish a procedure for Euthanasia for minors, but this regulation has raised serious doubts and has fueled the debate about its ethical, social, and legal implications.

KEYWORDS: euthanasia, palliative care, dignified death, terminally ill, Constitutional Court, autonomy of minors.

SUMARIO: I. INTRODUCCIÓN. II. DEL DERECHO A LA VIDA. II.1 La vida como valor ético y filosófico. II.2 Derecho a la vida de niños, niñas y adolescentes. III. DEFINICIÓN DE EUTANASIA. IV. DEL DERECHO A LA AUTODETERMINACIÓN FRENTE A LA EUTANASIA. IV.1 Límites del derecho a la autonomía en menores de edad. V. Los CUIDADOS PALIATIVOS.

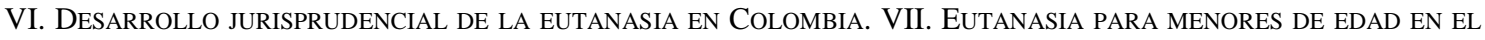
MUNDO. VIII. ENTREVISTA AL EX MINISTRO DE SALUd ALEJANDRO GAVIRIA URIBE, PONENTE DE LA RESOLUCIÓN QUE PROTOCOLIZÓ LA EUTANASIA PARA MENORES DE EDAD EN COLOMBIA. IX. REFLEXIONES FINALES. X. BibliOgRAFÍA.

\section{INTRODUCCIÓN}

El debate acerca de la eutanasia en Colombia ha estado presente desde hace muchos años, pero cuando ésta se refiere a menores de edad estamos hablando inevitablemente de una innovación constitucional, un acontecimiento que plantea adelantos en materia del derecho a morir dignamente, pero que a su vez propone un reto importante en el concepto de libertad individual para menores, derecho a la vida y moral social. 
Todas las decisiones públicas que se relacionen con el inicio de la vida o el final despiertan mucha controversia, sacan a la luz las pasiones y el fervor de la mayoría, y en el ágora de debate las opiniones son numerosas. Dworkin dice que este tipo de discusiones están permeadas por personajes más interesados en imponerse que en escuchar al adversario. Pero estos temas, más allá del componente religioso y el apasionamiento político, deben analizarse con seriedad desde lo académico, desde la ciencia jurídica y desde la bioética. Sobre todo, si lo evidente objetivamente es que se relativiza el derecho que le da sentido al Derecho como ciencia, la prerrogativa esencial del hombre: su propia vida.

La muerte digna se ha desarrollado en Colombia a partir la sentencia C-239 de 1997. Esta decisión judicial propuso una innovación constitucional muy trascendente, indicó que las libertades individuales pueden soslayar el derecho a la vida cuando la dignidad humana está siendo menoscabada por el dolor. Sin embargo, la evolución de los tiempos ha modificado la demanda de este procedimiento. Por mandato de la Corte Constitucional colombiana a través de la sentencia T-544 de 2017 se impuso al Ministerio de Salud la gran responsabilidad de establecer un protocolo para el ejercicio de la eutanasia en menores de edad. Así nace la resolución 825 de 2018 del Ministerio de Salud, que determina el trámite para el desarrollo del derecho a la muerte digna en niños, niñas y adolescentes (en adelante NNA).

Sin más antecedentes jurisprudenciales, sin un debate ético social, Colombia se convirtió en el tercer país del mundo, después de Bélgica y Holanda, en abrir la oportunidad para que se realicen procedimientos de eutanasia en menores de edad.

Por esto, se hace de vital importancia analizar las razones que motivaron el hecho de que se interpretara de manera extensa la autonomía de los NNA, se retara, de manera relativa, el concepto de patria potestad o autoridad de los padres e igualmente se procediera a desarrollar un derecho fundamental a través de un acto administrativo y no de una ley estatutaria.

\section{DEL DERECHO A LA VIDA}

La aprobación de la eutanasia para menores de edad supone un amplio análisis ético, filosófico, político y jurídico. Desde la perspectiva jurídica es importante señalar que la máxima de "no matar" ya encuentra su atenuación en el contexto normativo colombiano por virtud de diversas jurisprudencias. Es precisamente en esa medida donde se hace importante la reflexión acerca del derecho a la vida y sus connotaciones dentro del ordenamiento jurídico.

La vida, como máxima fundante del Estado Social de Derecho colombiano, se llega a comprender como elemento político cuando se estudia como una construcción del hombre en la historia y luego se normativiza en el Estado moderno como un derecho fundamental.

Colombia es un Estado Social de derecho y debido a su historia marcada por la violencia ha buscado siempre la protección de la vida desde todos sus ángulos. Conforme a ello, la Constitución Política de 1991 reconoce la vida como inviolable y le otorga la virtud de ser el primer derecho fundamental consagrado en nuestra Carta. El artículo 85 dice que el artículo 11, que contempla la vida como derecho fundamental, no requiere ampliación legislativa ya que es de aplicación inmediata, y de esta forma no precisa una ley que lo desarrolle. 


\section{II.1 La vida como valor ético y filosófico}

La vida humana puede ser analizada desde diversas perspectivas. Algunas de ellas relacionadas estrictamente con descubrir el valor espiritual, divino y trascendente del hombre. Otras, interesadas en encontrar su sentido ontológico, práctico, su deber ser.

La idea de sujeto viene de subjetividad, capacidad de ser y decidir. Y en ese sentido, se considera en primera medida que el hombre es conciencia, estado del ser. Y desde luego, ese ser, se hace distinto de los demás seres vivos a través de su capacidad de decidir.

La valía del hombre reposa en su virtud de ser único (intelectual y genéticamente), su capacidad de diferenciarse del otro. Aristóteles, Kant y Descartes, entre otros, han sido referentes de este pensamiento. El hombre vale en tanto es.

Para Jhon Stuart Mill (1948), desde una perspectiva utilitarista, lo más importante en un Estado es generar el máximo placer posible a sus ciudadanos. Hay que decirlo, ese placer aborda aspectos intelectuales, físicos, entre otros. Para Jhon Rawls (1995), desde el liberalismo, el ciudadano debe concebirse autónomo, capaz de decidir y desarrollar su proyecto de vida en tanto que el Estado no debe interferir sino garantizar el desarrollo de sus derechos en la medida que estos no afecten a los demás.

El dilema que se debe abordar desde la perspectiva ética y filosófica es si algunas vidas son, o pueden llegar a ser, más valiosas que otras. Si en algún momento o bajo determinadas circunstancias las formas de concebir la vida o los estados de inconsciencia pueden llegar a reducir el valor único de la vida. Si el hecho de considerar incapaz a un individuo, como se consideran lo menores de edad en Colombia, puede llegar a menoscabar su valor como persona.

Séneca decía: "la muerte es un castigo para alguno, para otros un regalo, y para muchos un favor". Kant lo expresó de dos formas particulares. En primera medida, decía que existe la dignidad ontológica. Aquella que se expresa en el valor del ser de manera intrínseca, profunda. Y luego, se refirió la dignidad moral, que dice que el hombre puede valorarse según sus acciones, y que estas pueden obedecer o no a la dignidad ontológica.

En últimas, debemos decir, que el valor de la vida desde la ética y la filosofía está vinculado a ver el hombre como ser o como acción, pero desde ambas perspectivas se erige la vida como elemento máximo de la ética, la filosofía, la sociología, la antropología y, desde luego, el Derecho.

\section{II.2 Derecho a la vida de niños, niñas y adolescentes}

Carlos A. Rezzonico (1999) afirma:

La vida y la salud humana tienen un valor intrínseco derivado de la misma dignidad de la persona humana. Por este carácter sagrado de la vida, cada persona, con prescindencia de su edad y características psicofísicas, tiene -sin exclusión- el mismo derecho básico a la vida y merece el respeto y la protección de la sociedad y de todos los profesionales dedicados a su cuidado. (P.1).

En el entendido de que los NNA cuentan con mayor protección y salvaguarda de su vida, se crea una postura contraria de derechos frente a su libre expresión, lo que hace que 
flaquee su autodeterminación al estar sujetos a las decisiones de sus representantes legales o apoderados, esto mengua de forma directa su voluntad.

Respecto de la priorización que se les da a los menores dentro de las normativas internacionales e internas de las naciones debemos hacer hincapié en la Convención de los Derechos del niño que tuvo lugar en New York el 20 de noviembre de 1989 la cual:

Reúne los derechos humanos de la infancia que estaban articulados en otros instrumentos internacionales. Esta Convención articula los derechos de un modo más completo y proporciona una serie de principios rectores que conforman el concepto fundamental que tenemos de la infancia. (Fondo de las Naciones Unidas para la Infancia [Unicef], s.f.).

Es vital definir que los niños tienen preferencia en el ejercicio de sus derechos y que el Estado debe ser garante de que estos se vean materializados, ya que las limitaciones que hemos divisado, fundamentalmente sobre la autonomía y la dignidad humana, no deben soslayar el derecho superior de la vida. Por el contrario, se debe proteger al menor, incluso si esto supone atenuar su autonomía.

\section{DEFINICIÓN DE EUTANASIA}

Hacer precisiones terminológicas acerca de la eutanasia es fundamental para estructurar un buen análisis. Sobre todo, si se tiene en cuenta que por lo general las personas crean definiciones cargadas de mentiras o medias verdades acerca de esta práctica. Definiciones que pueden llegar a ser peligrosas, como cuando se dice que eutanasia es el acto donde un médico mata a un paciente. Estas imprecisiones llegan a menoscabar las implicaciones conceptuales y sociales de la eutanasia y a desvalorizar la discusión.

Por esto es importante hacer uso de definiciones que nos dejen ver la realidad objetivamente.

En primera medida, desde su etimología, Eutanasia quiere decir buena muerte o buen

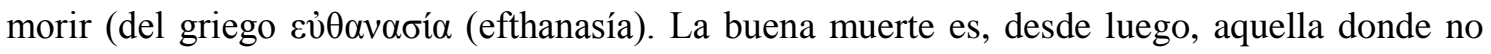
hay padecimientos tortuosos o sufrimiento, sino que se desciende a ella de manera neutra y tranquila. Así, decimos que la eutanasia es "la acción u omisión, por parte del médico u otra persona, con la intención de provocar la muerte del paciente terminal o altamente dependiente, por compasión y para eliminarle todo dolor.” (Cano, C. et al., 2008).

Fue Francis Bacon en el siglo XVII quien llevó el término a la medicina diciendo que el médico no sólo tiene funciones de hallar la cura de las enfermedades sino también de mitigar los sufrimientos que produce la misma.

\section{DEL DERECHO A LA AUTODETERMINACIÓN FRENTE A LA EUTANASIA}

Toda discusión acerca de la vida o la muerte pasa también por un análisis del hombre y sus libertades. La capacidad de decidir, de tomar nuestras propias determinaciones, nos hace seres pensantes. Esta cualidad encuentra su fundamento en el campo jurídico cuando hablamos del derecho a la autonomía o la autodeterminación. 
En el I Foro de eutanasia en niños, niñas y adolescentes el doctor Jhon Wilson Osorio afirma "La autonomía es asumir la vida como si fuera mía mi vida, y actuar conforme a mis propios designios considerando las consecuencias de mis actos en mí y para los otros". Universidad CES (2018).

Colombia es un país garante de la autonomía y las libertades individuales. Así, la vida se reconoce en nuestro ordenamiento jurídico como un derecho y no como un deber, y es cada ciudadano el titular de ese derecho.

Desde luego, la vida es un bien jurídico que el Estado busca proteger, pero cuando un ciudadano decide renunciar a ese derecho, el Estado no puede suprimir la libertad individual, su autonomía. Esta visión de la autonomía no puede radicalizarse, pues podríamos llegar a considerar la vida humana como simplemente una propiedad particular. Si así fuera todas las personas tendrían "derecho al suicidio" sin limitación de causales y supervisión estatal. Por el contrario, la sociedad tiene un interés legítimo en proteger la vida de todos sus miembros, inclusive si alguno de ellos considera que su vida no vale nada.

Cabe decir de manera concreta que la verdadera autonomía es aquella que sin perjuicio del tipo de decisión que sea, se ejerce en favor de la vida y la dignidad humana.

\section{IV.1 Límites del derecho a la autonomía en menores de edad}

Hasta la modernidad el "niño" como categoría socio-antropológica e histórica no existía. La infancia no solo era vista en la antigüedad como un estado de incapacidad sino, incluso, como una enfermedad que debía ser superada cuanto antes.

Para Platón el niño es un ser guiado por la parte irracional de su alma, sin capacidad de decidir y orientado por sus deseos ${ }^{1}$. Aristóteles (s.f) citado por Pereira, R. (2011) afirma: "los jóvenes pueden ser geómetras y matemáticos, y sabios en cosas de esta naturaleza y en cambio, no parece que puedan ser prudentes", en otras palabras, no parece que puedan ser autónomos.

Kant, avanza un poco más en su definición y es quien propiamente va a ocuparse de responder acerca de la capacidad de los menores dentro de la sociedad anteponiendo superioridad de los padres. Dice:

En la primera juventud nadie sabe qué fines podrán ofrecérsenos en la vida; por eso los padres tratan de que sus hijos aprendan muchas cosas [...] los padres olvidan por lo común de reformar y corregir el juicio de los niños sobre el valor de las cosas que pudieran proponerse como fines. (2007, P.42).

En palabras de Esperanza Cabrera (2011):

Hasta los inicios del siglo XVIII no se define la infancia como una etapa propia de la vida de los seres humanos, aunque en este periodo se carece de autonomía y se depende totalmente del adulto. Una constante, además del maltrato, es su no existencia, su discriminación y exclusión frente a toda decisión que le afecta. (P.7).

\footnotetext{
${ }^{1}$ Ver: PLATÓN. Leyes 672 b-c. Madrid: Centro de Estudios Constitucionales, Tomo I, 1983.) .
} 
Es Rousseau quien transforma este concepto y le da al niño una connotación social visible. Por primera vez en la historia se dignifica la posición del niño y aunque no se llega a considerar un ser autónomo, por lo menos se le ve como un ser con voluntad relativa. Un ser que está en proceso de desarrollo.

El debate sobre la eutanasia para menores de edad muestra los avances legales, civiles, éticos y bioéticos que la humanidad ha conquistado en occidente. Esta discusión 50 o 100 años atrás habría sido imposible porque conceptos como el de "infancia y autonomía" son consecuciones históricas, es decir, no son inmanentes, de siempre o eternos.

En ese orden de ideas, hay que decir que la discusión acerca de la eutanasia para menores es la discusión acerca de su autonomía.

¿Desde cuándo comienza a ejercer autonomía el hombre? ¿A partir de qué instante comienza a ser autónomo el individuo? ¿Es aplicable la premisa de "igual reconocimiento como persona ante la ley"?

Desde la realidad colombiana, en primera medida se debe considerar que el desarrollo de la autonomía en menores de edad tiene una connotación relativa. Cuando sus decisiones son sobre temas importantes como la propiedad privada, se requiere indefectiblemente de la autorización de su representante legal. Se considera que no tienen suficiente "experiencia de vida" para decidir por sí solos. Así, es obligación del Estado garantizar no solo el pleno ejercicio de sus derechos sino también el cuidado y la protección de su integridad en todos los aspectos, aunque esto menoscabe su autonomía, pues esta contingencia simplemente busca su tutela.

Decidir sobre un tema tan trascendental como lo es la muerte, en circunstancias de extrema vulnerabilidad, ya que nos referimos a un enfermo terminal, requerirá de una madurez mental, que incluso, en las circunstancias que expresa la Corte Constitucional es difícil de hallar.

En este punto es indispensable la experticia de los profesionales que pueden evaluar el nivel de desarrollo cognitivo de los NNA, que pueden determinar la mejor manera de darles información y que deben manejar la concurrencia con el consentimiento de ambos padres, que siempre será obligatorio. En los casos en los que la representación legal sea ejercida por otros individuos o que los NNA se encuentren bajo la protección del Estado, la valoración del consentimiento sustituto deberá ser estricta. (Corte Constitucional de Colombia, Sala Quinta. (25 de agosto de 2017) [MP Gloria Díaz], Sentencia T-544).

En lo que concierne a la resolución 825 de 2018 del Ministerio de Salud, que reguló la eutanasia para menores en Colombia, la expresión que desarrolla de manera clara la autonomía se encuentra en el avance del artículo 2, donde se menciona que la toma de decisiones de los menores en el aspecto médico debe cumplir cuatro requerimientos: (i) capacidad de comunicar la decisión, (ii) capacidad de entendimiento, (iii) capacidad de razonar y (iv) capacidad de juicio.

La consideración de la Corte Constitucional en la sentencia T-544 de 2017, en primera medida, y del Ministerio de Salud en la resolución, acerca de la autonomía de los menores, propone un reto constitucional del cual no podemos pasar inadvertidos. La conciencia moral acerca de la muerte, ¿sería suficiente argumento para soslayar el derecho a la vida? El 
Ministerio ha expuesto de manera explícita la conciencia de los menores acerca de la muerte según el rango de edad.

De 0 a 3 años. La muerte no es un concepto real ni formal. No existe idea de muerte propia. De 3 a 6 años. El niño o niña desarrolla su pensamiento prelógico, intuitivo, aparece la idea de muerte como fenómeno temporal, la muerte es reversible o transitoria. No se consolida idea de muerte propia. De 6 a 12 años. El niño o niña desarrolla un pensamiento lógico, operacional, que le permite adquirir elementos que hacen parte del concepto de muerte, como la inmovilidad, universalidad e irrevocabilidad.[...] A partir de los 12 años. Desde los doce años de edad se presenta el concepto de muerte vinculado a la capacidad de abstracción desde el cual se logra el entendimiento de que todo el mundo, incluso uno mismo, va a morir, tarde o temprano. Ministerio de Salud de Colombia, 2018, Artículo 2 [Capítulo I] Resolución 825.

Finalmente, con todo y el consentimiento de los padres, y aún con la aprobación de los menores, el deber jurídico y superior del Estado de proteger a los NNA en su integridad y velar por el amparo de sus derechos fundamentales no desaparece.

\section{LOS CUIDADOS PALIATIVOS}

Cuando se habla de cuidados paliativos se hace referencia principalmente a aquellos cuidados o atenciones de carácter médico que se brinda al paciente que se encuentra en etapas terminales y sufre dolores insoportables a partir de las consecuencias que trae consigo la enfermedad. Estos son medicamentos que al ser suministrados facilitan el paso a la muerte dándole tranquilidad y reduciendo el sufrimiento a los mínimos efectos posibles. Además de esto, los cuidados paliativos no se reducen solo a medios de minimizar síntomas físicos, sino también a la ayuda de carácter psicológico y espiritual del paciente por la difícil transición de saber que se acerca el final de la vida en cualquier momento.

\section{DESARROLLO JURISPRUDENCIAL DE LA EUTANASIA EN COLOMBIA}

El origen del derecho a la muerte digna en Colombia, como ha pasado con el desarrollo de los temas que proponen transformaciones sociales complejas en nuestro país, ha estado en manos de la Corte Constitucional. Hasta 1997 la Eutanasia solo era un debate ético en el ágora social, pero la apremiante necesidad de regular y expandir la interpretación de las libertades individuales hizo que se transformara en una sentencia judicial, la C-239 de 1997. Sentencia que se ha convertido en un hito de la bioética en el país y que tuvo como magistrado ponente al maestro e ilustre jurista Carlos Gaviria Díaz (QEPD).

En palabras del ex ministro de salud Alejandro Gaviria Uribe (2018):

"La Corte Constitucional ha tenido un papel preponderante en este, y otros asuntos similares, que involucran valores y creencias de la sociedad (...) Más allá de la defensa de los derechos humanos, la Corte ha promovido el cambio cultural $\mathrm{y}$ ha contribuido decididamente a transformar los modos de pensamiento de los colombianos." (P.195). 
Sobre la Eutanasia no podemos decir que exista una línea jurisprudencial definida, sino que hay una línea de decisión. Hay algunos fallos, pero ellos nos demuestran que no hay como tal una unificación de criterios sino atisbos y aportes en la creación de un derecho.

El último pronunciamiento de la Corte Constitucional al respecto de la muerte digna en Colombia lo encontramos en la sentencia T-544 de 2017. Esta sentencia presenta a través de sus páginas lo que podría ser visto como un avance constitucional o un exceso interpretativo de la Corte, otorgándole un alcance a la muerte digna que toca las fibras más sensibles de la sociedad pues se refiere a los menores de edad.

La Corte Constitucional reconoce que frente a los NNA hay un vacío normativo. Este reconocimiento es muy valioso porque indica que los menores de edad no van a ser cobijados a través de la regulación a mayores sino que el Ministerio de Salud debe, a través de un nuevo acto administrativo, garantizar la muerte digna a menores de edad teniendo en cuenta las características especiales de los derechos de los NNA.

La Corte hace de nuevo una exhortación al Congreso de la República, máximo legislador, para reglamentar a través de una ley el derecho a la muerte digna y le da un plazo de 2 años para cumplir ese fin.

Estudiar las razones que dan origen a la protocolización de la eutanasia para menores en el país no tomaría mucho tiempo. Bastaría leer la sentencia T-544 de 2017 y conocer lo planteado por la C-239 de 1997. Esta ausencia de profundidad académica, de debate social, genera incertidumbre jurídica y falta de legitimidad en la nueva normatividad.

Es de trascendental importancia transitar el camino del debate social con la resolución 825 del Ministerio de Salud. Sobra decir que no es una discusión menor.

La eutanasia, para buena parte de la población, puede resultar inadmisible en todas las formas que se presente y para todas las edades. Para ese grupo de ciudadanos el debate está cerrado en cuanto a la eutanasia en menores de edad. Sin embargo, no todos los que comparten el ejercicio de la eutanasia para mayores de edad compartirían la permisibilidad de este procedimiento para menores de edad. Bajo distintos argumentos, uno de ellos la significación de la vida o muerte que mentalmente podrían tener los menores.

"Para considerar o no éticamente aceptable la eutanasia infantil se requiere asumir antes una visión sobre el valor de la vida del niño, aun en condiciones de enfermedad y sufrimiento. Se trata de una pregunta metafísica sobre el significado de la vida, ante la cual no hay respuestas universales y por tanto, quienes deciden ante casos particulares no pueden imponer sus propias creencias." (Beca, J. P., \& Leiva, A. ,2014 P.4).

Si son incapaces para tomar decisiones cotidianas, si requieren el acompañamiento de su representante legal para diversas situaciones de la vida diaria, el interrogante es cómo a la luz de los requerimientos de esta nueva norma encuentran la posibilidad de solicitar su propia muerte.

Desagregar la vida de los niños, niñas y adolescentes en el país de su sentido más profundo, de la protección sagrada por parte del Estado, puede tener consecuencias nefastas que derivarían a la postre en desvalorización de la vida, menoscabo de su significado ontológico. 


\section{EUTANASIA PARA MENORES DE EDAD EN EL MUNDO}

Bélgica ha sido pionero en torno al tema de la muerte asistida para enfermos terminales enfocado a los NNA, abordando esta materia a lo largo de su historia con el fin de regularla y ampliar los diferentes documentos internacionales que comprenden la eutanasia. Desde enero de 2001 se han dado unos pasos decisivos para la aprobación de la eutanasia. Una comisión del Senado aprobó uno de los artículos principales del proyecto de ley que pretende la despenalización de la «dulce muerte».

Holanda también ha presentado gran iniciativa en lo que atañe a la eutanasia, siendo el primero en legalizarla en el mundo para mayores de edad. Este país también regula en cierta medida la eutanasia aplicada a los NNA, pero esta regulación es estricta en lo que se refiere a los límites de edad, puesto que:

No autoriza en ningún caso la eutanasia en menores de 12 años. Pero sí la autoriza en mayores de 12 y menores de 16 años, siempre y cuando los padres presten su autorización en forma expresa, y en mayores de 16 y menores de 18 años, simplemente oyendo a los padres. (Beca, J. P., \& Leiva, A., 2014 P.2).

\section{ENTREVISTA AL EX MINISTRO DE SALUD ALEJANDRO GAVIRIA URIBE, PONENTE DE LA RESOLUCIÓN QUE PROTOCOLIZÓ LA EUTANASIA PARA MENORES DE EDAD EN COLOMBIA}

Dayron Reyes: ¿Qué significa, para un funcionario público, tomar decisiones impopulares, decisiones que tienen un golpe mediático tan fuerte como la eutanasia para menores de edad?

Alejandro Gaviria: No fue fácil. En este tema, en particular, existe una brecha muy grande entre la jurisprudencia (las instituciones formales) y los valores y creencias de la sociedad. Traté de hacerlo con ponderación, pero debo reconocer que estamos lejos de una comprensión adecuada del tema. Me sorprendió, por ejemplo, la oposición vehemente de muchos paliativitas.

DR.: Lichtenberg decía que la razón siempre es más polémica que dogmática, ¿usted considera que la lucha por la muerte digna en Colombia, con todo el dogmatismo que respira el país, ha valido la pena darla?

A.G.: Yo creo que sí. Esta lucha permitió un debate más amplio e intenso sobre la muerte digna, la obstinación terapéutica y la excesiva medicalización de la muerte. No es un asunto resuelto. El proceso de cambio es necesariamente incremental. Pero considero que avanzamos más rápido en los últimos cinco años que en los previos treinta.

D.R: Colombia es el tercer país del mundo en regular la eutanasia para menores de edad, después de Bélgica y Holanda, usted considera éste hecho como una evolución o una involución constitucional.

A.G.: Es una evolución. Pero debo reconocer al mismo tiempo que la Corte actuó de manera apresurada. Me habría gustado que el tema se tratará en sala plena y que se hubiera citado a una audiencia pública. La legitimidad viene de la discusión. Pero al Corte muchas veces parece ajena a estos asuntos. Yo he señalado un contraste entre la sentencia 
que despenalizó el aborto (en este caso hubo una discusión muy larga antes de la sentencia) y la sentencia de eutanasia en menores (en este caso no hubo discusión).

D.R.: La sentencia T-544 de 2017 es la que da origen a la resolución 825 de 2018. Cómo afronta usted esta tarea desde el Min Salud.

A.G.: Teníamos un grupo de bioeticistas en el Ministerio. Trabajamos inicialmente con ellos. Después abrimos la discusión, citamos a un grupo de expertos, médicos, enfermeras, paliativitas, expertos en bioética, de diversos origines y posturas. Discutimos con ellos largamente. Recogimos las sugerencias. Produjimos un primer borrador. Y después los volvimos a citar a trabajar ya en concreto sobre el borrador. Así se construyó la resolución.

D.R.: La consideración de la Corte Constitucional, en primera medida, y luego del Ministerio de Salud, acerca de la autonomía de los menores, propone un reto constitucional del cual no podemos pasar inadvertidos. La conciencia moral acerca de la muerte, como lo proponen la Corte y el Ministerio, ¿sería suficiente argumento para soslazar el derecho a la vida?

A.G.: La resolución es muy enfática en el tema de la autonomía. Invierte la carga de prueba. Un grupo de expertos debe decidir si el menor de edad es efectivamente consciente de la inexorabilidad de la muerte, de lo que está ocurriendo. En últimas, solo aplicaría a menores que tenga un nobel de raciocinio tal que puedan ser considerados adultos.

D.R.: ¿Usted considera que esta es una resolución de cuidados paliativos?

A.G.: Sí, más que una resolución de eutanasia es de cuidados paliativos. Así la concebimos, ese es el espíritu esencial de la norma.

\section{REFLEXIONES FINALES}

Investigar sobre la eutanasia para menores de edad en Colombia, ha buscado situar la discusión acerca de la muerte digna en este país sobre los asuntos más relevantes, el análisis del componente jurídico y no únicamente la perspectiva moral. Desde luego, la construcción de nuevas realidades en la modernidad supone comprender la moral social, pero partimos de mencionar que sólo a través de innovaciones jurídicas las sociedades pueden estudiar, modificar o revaluar sus conductas.

Así, el panorama desarrollado por los filósofos antiguos y de la ilustración cobra sentido. El valor del hombre es el hombre mismo.

En esa medida, el hombre es también el peso de su propia conducta, el peso de su libertad. Y esta interpretación se extiende hacia los menores de edad, que por muchos años en la historia fueron marginados y hoy se perciben como sujetos de especial protección por parte del Estado otorgándoles a su vez la capacidad relativa para tomar decisiones.

En Colombia el debate de la eutanasia para menores de edad no está cerrado, de hecho, apenas comienza. Desde este artículo hemos querido abordar sus implicaciones haciendo un análisis jurídico y bioético de cómo la relativización, la sublevación de la autonomía y la ponderación de las libertades podría terminar afectado a su vez los derechos de los menores. 
Abordar la dignidad humana como un derecho ambivalente, que es usado tanto para defender la eutanasia como para estar en contra de ella, significa no sólo ver este derecho como correlación del derecho a la vida sino también replantear el hecho de que un enfermo terminal, por el hecho de serlo, se considerarse con un nivel de vida indigno.

Según nuestra investigación, la mejor expresión de respeto hacia la vida es el cuidado de ésta en sus últimas manifestaciones. En esa medida, los cuidados paliativos se presentan como la única perspectiva moralmente aceptable y jurídicamente viable para ayudar a morir a los menores de edad.

Si bien, el desarrollo jurisprudencial acerca de la eutanasia para menores es corto, también cabe decir que la resolución 825 del Ministerio de Salud siembra serias dudas y no resuelve el problema por completo. De hecho, podría estar creando más problemas.

Cabe decirlo, el Ministerio de Salud tomó el tema con rigor al establecer un procedimiento que pudiese llenar las expectativas de lo propuesto en la sentencia de constitucionalidad T-544 de 2017, pero no es directamente quien debe resolver el tema en cuestión. Es el Congreso de la República quien debe entrar a regular este tema para legitimarlo en la ciudadanía.

Según nuestro análisis, las preguntas que debe elaborar la sociedad civil no pueden estar planteadas desde un minimalismo moral. Es decir, el interrogante no es: ¿está de acuerdo con la eutanasia para menores de edad? Sino ¿cuándo la eutanasia para menores puede ayudar al desarrollo social? $\mathrm{O}$ ¿cuándo las implicaciones de este procedimiento pueden violentar los derechos de los más vulnerables?

Este tipo de situaciones jurídicas nos hacen olvidar del principio mismo del Derecho, pues lo que le da sentido a la vida, y en especial a la vida de quienes no pueden defender su propia existencia, es la expresión de morir descendiendo a los brazos del silencio y no la acción agitada de una inyección letal.

\section{BIBLIOGRAFÍA}

- Platón. Leyes 672 b-c. Madrid: Centro de Estudios Constitucionales, Tomo I, 1983.

- A. Gaviria (2016). Alguien tiene que llevar la contraria. Colombia. Editorial Ariel.

- A. Gaviria (2018). Hoy es siempre todavía. Colombia. Editorial Ariel.

- Aguilar Cavallo, G. (2008). "El principio del interés superior del niño y la Corte Interamericana de Derechos Humanos". Estudios Constitucionales, 6 (1).

- BeCA, J. P., \& LeIVA, A. (2014). “Podría ser aceptable la eutanasia infantil?”, Revista chilena de pediatría, 85 (5), 608-612.

- CORTE COnStituCiOnAl De COlOMBiA, Sala Plena. (20 de mayo de 1997). Sentencia C-239. [Mp Carlos Gaviria Díaz].

- CORTE CONSTITUCIONAL DE COLOMBIA, Sala Quinta de Revisión. (25 de agosto de 2017) Sentencia T-544. [Mp Gloria Stella Ortíz Delgado].

- ESPAÑol, U. C. (2016). Convención sobre los Derechos del Niño. FUNDACIÓN UNICEFCOMITÉ ESPAÑOL. 
- GutiérRez, J. V. (2007). "La práctica de la eutanasia en Bélgica y la «pendiente resbaladiza»". Cuadernos de bioética, 18 (1), 71-87.

- Humanos, D. (1948). Declaración Universal de los Derechos humanos. La Convención Internacional de los Derechos del Niño. Naciones Unidas. Declaración sobre la Protección de todas las personas contra la tortura.

- Kant, E. (2007). Fundamentación de la metafísica de las costumbres. Edición de Pedro M. Rosario Barbosa.

- Manzano, M. R. Discusión jurídica frente a los alcances del procedimiento para hacer efectivo el derecho a morir con dignidad de los niños, las niñas y los adolescentes en Colombia.

- MiLl, S. (1984). John: El utilitarismo. Alianza: Madrid.

- Ministerio de SALUd y PROteCCIÓN Social. 2018. Resolución 825.

- Nombela Cano, C., López Timoneda, F., Serrano Ruiz-Calderón, J. M., Postigo Solana, E., Abellán Salort, J. C., \& Prensa Sepúlveda, L. (2008). "La eutanasia: perspectiva ética, jurídica y médica". Observatorio de Bioética. Obtenido de http://www. observatoriobioetica. org/wp-content/uploads/2013/12/La-eutanasia.-Perspectiva-\% C3\% A9tica-jur\% C3\% ADdica-ym\% C3\% A9dica. pdf.

- PRADA URIBE, S. Fundamentos éticos para repensar la normatividad colombiana frente a solicitudes de eutanasia desde el pensamiento Rawlsiano Steven.

- RAWLS, J. (1995). Liberalismo político. UNAM.

- Rezzónico, C. A. (2004). "Bioética y derechos de los niños". Archivos argentinos de pediatría, 102 (3), 214-219.

- SÁNCHEZ, E. P. (2015). "Eutanasia, autonomía y la libre disponibilidad de la propia vida", Astrolabio, (15), 303-333.

- TocQueville, A. DE (1984). La democracia en América. Madrid: SARPE.

- UNIVERSIDAD CES (06 de marzo de 2018). "I Foro de eutanasia en niños, niñas y adolescentes". [Archivo de video] Recuperado de https://bit.ly/2Uu6acm

- Villegas, G. L. (2001). "Eutanasia Activa en Colombia: Algunas Reflexiones sobre la Jurisprudencia Constitucional”, La. Rev. Derecho del Estado, 11, 95. 